Chinese Journal of Organic Chemistry

NOTE

\title{
白喉乌头中二萜生物碱及其拒食活性研究
}

\author{
陈 琳 ${ }^{a, b, c}$ 王 倩 ${ }^{a}$ 黄 帅 ${ }^{a}$ 单连海 ${ }^{a}$ 高 峰 ${ }^{a}$ 周先礼 $*, a, b$ \\ ( ${ }^{a}$ 西南交通大学生命科学与工程学院 成都 610031) \\ ( ${ }^{b}$ 西南交通大学材料科学与工程学院 成都 610031) \\ ( ${ }^{c}$ 西华师范大学化学化工学院 南充 637002)
}

\begin{abstract}
摘要 从白喉乌头(Aconitum leucostomum Vorosch.)中分离得到 13 个二萜生物碱, 采用 HR-ESI-MS, 1D NMR 和 2D $\mathrm{NMR}$ 等波谱技术确定了它们的结构. 其中一个为新的 $\mathrm{C}_{18}$ 二萜生物碱: 刺乌宁, 其余为已知化合物. 同时考察了其中 12 个化合物对甜菜夜蛾 3 龄幼虫的拒食活性, 结果表明化合物氨茴酰基牛扁碱和东乌头定的活性较好 $\left(\mathrm{EC}_{50}<1\right.$ $\left.\mathrm{mg} / \mathrm{cm}^{2}\right)$, 其次是 $N$-acetylsepaconitine, 赣乌碱和 $N$-去乙酰高乌甲素 $\left(\mathrm{EC}_{50}<2 \mathrm{mg} / \mathrm{cm}^{2}\right)$.
\end{abstract}

关键词 乌头属; 白喉乌头; 二萜生物碱; leucostonine; 拒食活性

\section{Diterpenoid Alkaloids from Aconitum leucostomum and Their Antifeedant Activity}

\author{
Chen, Lin ${ }^{a, b, c} \quad$ Wang, Qian $^{a} \quad$ Huang, Shuai $^{a} \quad$ Shan, Lianhai ${ }^{a}$ \\ Gao, Feng $^{a} \quad$ Zhou, Xianli*,a,b \\ ( ${ }^{a}$ School of Life Science and Engineering, Southwest Jiaotong University, Chengdu 610031) \\ ( ${ }^{b}$ School of Material Science and Engineering, Southwest Jiaotong University, Chengdu 610031) \\ ( ${ }^{c}$ School of Chemistry and Chemical Engineering, China West Normal University, Nanchong 637002)
}

\begin{abstract}
A new $\mathrm{C}_{18}$ diterpenoid alkaloid, leucostonine (1), was isolated from the whole plant of Aconitum leucostomum Vorosch., together with twelve known diterpenoid alkaloids. Their structures were elucidated on the basis of extensive spectroscopic analysis, including HR-ESI-MS, 1D NMR and 2D NMR spectra. 12 compounds were tested for their antifeedant activity against larvae of Spodoptera exigua Hiibner. Anthranoyllycoctonine and avadharidine showed considerable potent antifeedant activity $\left(\mathrm{EC}_{50}<1 \mathrm{mg} / \mathrm{cm}^{2}\right)$, followed by $N$-acetylsepaconitine, finaconitine and $N$-deacetylappaconitine $\left(\mathrm{EC}_{50}<2\right.$ $\mathrm{mg} / \mathrm{cm}^{2}$ ).

Keywords aconitum; Aconitum leucostomum; diterpenoid alkaloid; leucostonine; antifeedant activity
\end{abstract}

二萜生物碱由于其结构的复杂多样性，同时又具有 消炎、镇痛、抗心律失常、抗真菌、抗癌以及杀虫和拒 食等多种生物活性而越来越受到人们的关注, 其主要分 布在乌头属、翠雀属和飞燕草属中 ${ }^{[1]}$. 白喉乌头 (Aconitum leucostomum Vorosch.) 为毛茛科乌头属植物, 主要分布于中国新疆、甘肃西北部(山丹), 哈萨克斯坦 也有分布 ${ }^{[2]}$. 据《哈萨克药志》记载, 白喉乌头在哈萨克 民族作为草乌的代用品使用, 其味辛、热、苦、大毒, 有
祛风除湿、温经止痛的功能，常用于风寒湿痹等疾病， 为西北少数民族常用民间草药之一. 对白喉乌头中的化 学成分研究已有报道 ${ }^{[3]}$, 本课题组对白喉乌头中的药用 成分进一步研究, 分离得到 13 个二萜生物碱, 用 HRESI-MS、IR、1D NMR 和 2D NMR 等波谱技术以及与 文献对比等方法确定了它们的结构(图 1), 其中一个为 新的二萜生物碱 leucostonine (1), 另外 12 个已知化合物 包括 7 个 $\mathrm{C}_{18}$ 型二萜生物碱: acosepticine (2), 喉乌碱

\footnotetext{
* Corresponding author. E-mail: zhouxl@swjtu.edu.cn Received February 16, 2017; revised March 9, 2017; published online April 1, 2017.

Project supported by the National Natural Science Foundation of China (No. 81402803), the Small Molecule Natural Medicine Innovation Team (No. 15TD0048), the Research Foundation for Educational Commission of Sichuan Province (No. 15ZB0140) and the Fundamental Research Funds for Central Universities (No. 2682017QY04).

国家自然科学基金(No. 81402803)、四川省小分子天然药物创新团队(No. 15TD0048)、四川省教育厅项目(No. 15ZB0140)、中央高校基本科研业务费 专项(No. 2682017QY04)资助项目.
} 
(leucostine，3)，刺乌定（lappaconidine，4)，刺乌宁 (lapaconine, 5), $N$-acetylsepaconitine (6), 赣 乌碱 (finaconitine, 7) 和 $N$-去乙酰高乌甲素 ( $N$-deacetylappaconitine, 8), 4 个 $\mathrm{C}_{19}$ 型二萜生物碱: acosanine (9), delphatine (10), 氨茴酰基牛扁碱(anthranoyllycoctonine, 11) 和东乌头定 (avadharidine, 12), 以及一个 $\mathrm{C}_{20}$ 型二萜生物 碱裸翠雀亭(denudatine, 13). 同时考察了其中 12 个化合 物对甜菜夜蛾幼虫的拒食活性.

\section{1 结果与讨论}

化合物 1 为白色固体, 碘化铋钾显阳性; 通过 HRESI-MS 图谱可知该化合物分子式为 $\mathrm{C}_{22} \mathrm{H}_{35} \mathrm{NO}_{5}(\mathrm{~m} / \mathrm{z}$ : $394.2584[\mathrm{M}+\mathrm{H}]^{+}$, calcd for 394.2593). IR 图谱显示化合 物 1 中存在羟基 $\left(3392 \mathrm{~cm}^{-1}\right)$. 结合化合物 1 的 ${ }^{1} \mathrm{H}$ NMR、 ${ }^{13} \mathrm{C} \mathrm{NMR}$ 图谱和 DEPT 谱, 表明其含有一个氮乙基 $\left[\delta_{\mathrm{H}}\right.$ $\left.1.09(\mathrm{t}, J=7.2 \mathrm{~Hz}, 3 \mathrm{H}) ; \delta_{\mathrm{C}} 50.6 \mathrm{t}, 13.9 \mathrm{q}\right]$, 两个甲氧基 $\left[\delta_{\mathrm{H}}\right.$ $\left.3.40(\mathrm{~s}, 3 \mathrm{H}), \delta_{\mathrm{C}} 57.8 \mathrm{q} ; \delta_{\mathrm{H}} 3.35(\mathrm{~s}, 3 \mathrm{H}), \delta_{\mathrm{C}}(56.4 \mathrm{q})\right]$. 进一 步分析 ${ }^{13} \mathrm{C} N M R$ 与 DEPT 图谱可知化合物 1 中还有 18 个碳原子, 分别是 6 个亚甲基 $\left(\delta_{\mathrm{C}} 24.0,29.4,29.9,36.4\right.$, 37.3 和 53.6), 9 个次甲基 $\left(\delta_{\mathrm{C}} 33.7,37.1,40.4,43.3,47.0\right.$, $65.0,72.6,82.8$ 和 85.0) 和 3 个季碳 $\left(\delta_{\mathrm{C}} 49.2,77.4\right.$ 和 86.2), 推测该化合物应为 $\mathrm{C}_{18}$ 型二萜生物碱 $\left[{ }^{[4]} \cdot{ }^{13} \mathrm{C} \mathrm{NMR}\right.$ 数据可 知化合物 1 中含有五个含氧取代的碳信号, 除两个甲氧 基以外, 推测化合物 1 中还含有 3 个羟基. $\mathrm{HMBC}$ 图谱 (图 2)中, 两个甲氧基信号 $\left[\delta_{\mathrm{H}} 3.40,3.35\right.$ (each s, 3H)]分 别与 C-14 $\left(\delta_{\mathrm{C}} 85.0\right)$ 和 C-16 $\left(\delta_{\mathrm{C}} 82.8\right)$ 有远程相关, 可知 2

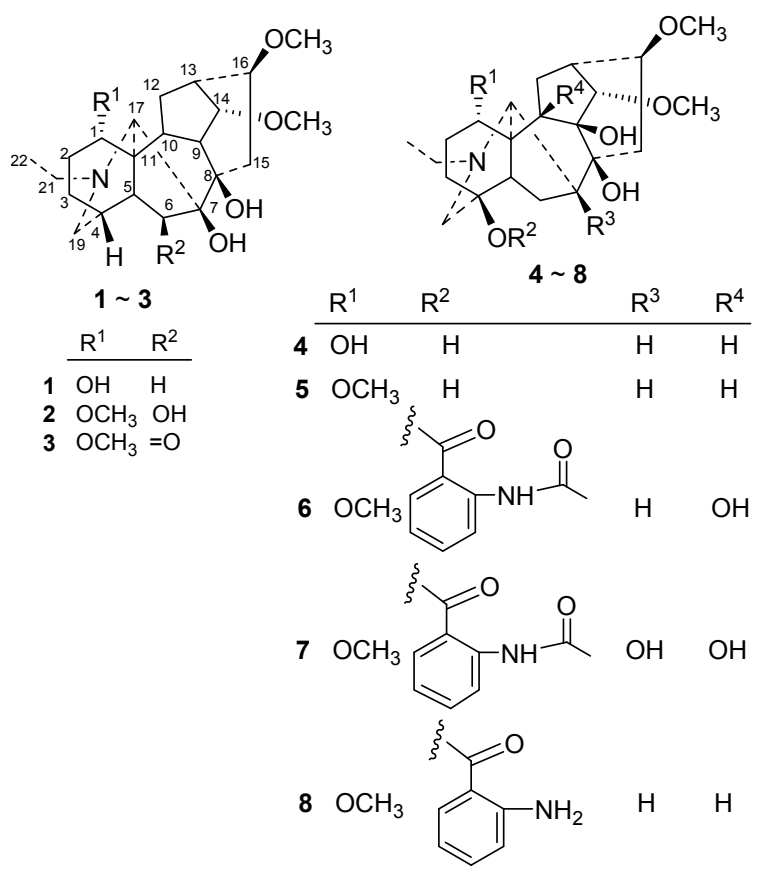

个甲氧基分别位于 C-14 和 C-16 位. 分析 HMBC 图谱, $\mathrm{H}-2\left(\delta_{\mathrm{H}} 1.55\right)$ 和 $\mathrm{H}-5\left(\delta_{\mathrm{H}} 1.85\right)$ 与 C-1 $\left(\delta_{\mathrm{C}} 72.6\right)$ 有相关, 同 时, 在 ${ }^{1} \mathrm{H}$ NMR 中 $\mathrm{H}-1$ ( $\left.\delta_{\mathrm{H}} 3.64\right)$ 为一个宽单峰且 $\mathrm{C}-1$ 的 核磁数据为 $\delta_{\mathrm{C}} 72.6$, 可知 C-1 连有一个 $\alpha$ 构型的羟基 ${ }^{[5]}$. 进一步分析 HMBC 图谱, H-6 $\alpha\left(\delta_{\mathrm{H}} 1.43\right)$ 和 H-15 $\alpha\left(\delta_{\mathrm{H}}\right.$ $2.92)$ 与 C-7 $\left(\delta_{\mathrm{C}} 86.2\right)$ 有相关, H-6 $\left(\delta_{\mathrm{H}} 1.43 ; \delta_{\mathrm{H}} 2.43\right), \mathrm{H}-10$ $\left(\delta_{\mathrm{H}} 1.79\right)$ 和 H-14 $\left(\delta_{\mathrm{H}} 3.72\right)$ 与 C-8 $\left(\delta_{\mathrm{C}} 77.4\right)$ 有远程相关, 可 以确定余下的两个羟基位于 C-7 和 C-8 位. NOESY 图谱 中 H-1 和 H-10, H-10 和 H-14, H-16 和 H-17 有相关, 可 以进一步确定 C-1 的羟基应该为 $\alpha$ 构型, C-14 位甲氧基 为 $\alpha$ 构型, 而 C-16 位甲氧基为 $\beta$ 构型. 综上所述, 化合 物 1 的结构鉴定为 leucostonine (1) (图 1).

化合物 2 13 为已知化合物，经波谱分析与文献数 据对照, 将它们鉴定为 acosepticine $(2)^{[6]}$, 喉乌碱 $(3)^{[7]}$, 刺乌定 $(4)^{[8]}$, 刺乌宁 $(5)^{[9]}, N$-acetylsepaconitine $(\mathbf{6})^{[10]}$, 赣 乌碱(7) ${ }^{[11]}, N$-去乙酰高乌甲素 $(\mathbf{8})^{[12]}$, acosanine $(9)^{[12]}$, delphatine $(\mathbf{1 0})^{[13]}$, 氨茴酰基牛扁碱 $(\mathbf{1 1})^{[14]}$, 东乌头定 $(\mathbf{1 2})^{[15]}$ 和裸翠雀亭 $(13)^{[16]}$. 其中, 化合物 2, 4 7, 9, 12 和 13 为首次从该植物中分离得到.

采用选择性叶碟法考察了白喉乌头中 7 个 $\mathrm{C}_{18}, 4$ 个 $\mathrm{C}_{19}$ 和 1 个 $\mathrm{C}_{20}$ 二萜生物碱对甜菜夜蛾(Spodoptera exigua Hiibner) 3 龄幼虫的拒食活性, 以乌头碱为对照, 结果见 表 2. 从表中可以看出, $\mathrm{C}_{18}$ 二萜生物碱中, 活性最好的 化合物分别是 6,7 和 $8\left(\mathrm{EC}_{50}<2 \mathrm{mg} / \mathrm{cm}^{2}\right)$, 其次是 2,4 和 $1\left(\mathrm{EC}_{50}=8 \sim 21 \mathrm{mg} / \mathrm{cm}^{2}\right)$, 化合物 3 基本没有活性 $\left(\mathrm{EC}_{50}>30 \mathrm{mg} / \mathrm{cm}^{2}\right)$; 这也是首次对 $\mathrm{C}_{18}$ 型二萜生物碱的
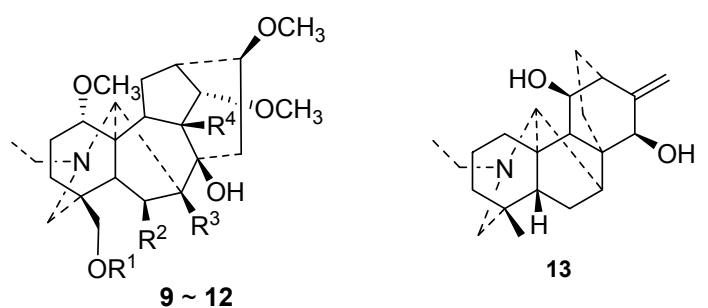

\begin{tabular}{lllll} 
& $\mathrm{R}^{1}$ & $\mathrm{R}^{2}$ & $\mathrm{R}^{3}$ & $\mathrm{R}^{4}$ \\
\hline $\mathrm{CH}_{3}$ & $\mathrm{OH}$ & $\mathrm{OH}$ & $\mathrm{H}$ \\
& $\mathrm{CH}_{3}$ & $\mathrm{OCH}_{3}$ & $\mathrm{OH}$ & $\mathrm{H}$
\end{tabular}<smiles>CC(=O)c1ccccc1N</smiles>
$\mathrm{OCH}_{3} \mathrm{OH} \quad \mathrm{H}$ 12<smiles>COC(=O)c1ccccc1NC(=O)CCC(N)=O</smiles>

图 1 从白喉乌头中分离得到的二萜生物碱

Figure 1 Diterpenoid alkaloids isolated from Aconitum leucostomum 
表 1 化合物 1 的核磁共振图谱数据(400 MHz for ${ }^{1} \mathrm{H} \mathrm{NMR}, 100 \mathrm{MHz}$ for ${ }^{13} \mathrm{C} \mathrm{NMR}, \mathrm{CDCl}_{3}$ )

Table 1 NMR spectroscopic data of compound 1 (400 MHz for ${ }^{1} \mathrm{H}$ NMR, $100 \mathrm{MHz}$ for ${ }^{13} \mathrm{C} \mathrm{NMR}, \mathrm{CDCl}_{3}$ )

\begin{tabular}{|c|c|c|c|c|c|}
\hline No. & ${ }^{1} \mathrm{H}$ NMR & ${ }^{13} \mathrm{C}$ NMR & No. & ${ }^{1} \mathrm{H}$ NMR & ${ }^{13} \mathrm{C}$ NMR \\
\hline 1 & 3.64 br.s & $72.6 \mathrm{~d}$ & 13 & $2.43^{a}$ & $37.1 \mathrm{~d}$ \\
\hline 2 & $1.55^{a}(\alpha, \beta)$ & $29.4 \mathrm{t}$ & 14 & $3.72 \mathrm{t}(4.8)$ & $85.0 \mathrm{~d}$ \\
\hline 3 & $\begin{array}{l}1.61^{a}(\beta) \\
1.98^{a}(\alpha)\end{array}$ & $24.0 \mathrm{t}$ & 15 & $\begin{array}{l}1.61^{a}(\beta) \\
2.92^{a}(\alpha)\end{array}$ & $36.4 \mathrm{t}$ \\
\hline 4 & $1.98^{a}$ & $33.7 \mathrm{~d}$ & 16 & $3.26 \mathrm{t}(8.4)$ & $82.8 \mathrm{~d}$ \\
\hline 5 & $1.85 \mathrm{~m}$ & $40.4 \mathrm{~d}$ & 17 & 2.63 br.s & $65.0 \mathrm{~d}$ \\
\hline 6 & $\begin{array}{l}1.43 \mathrm{~d}(14.4)(\alpha) \\
2.43^{a}(\beta)\end{array}$ & $37.3 \mathrm{t}$ & 19 & $\begin{array}{l}2.53 \mathrm{~d}(11.2)(\alpha) \\
3.16 \mathrm{dd}(4,11.2)(\beta)\end{array}$ & $53.6 \mathrm{t}$ \\
\hline 7 & - & $86.2 \mathrm{~s}$ & 21 & $2.92^{a}$ & $50.6 \mathrm{t}$ \\
\hline 8 & - & $77.4 \mathrm{~s}$ & 22 & $1.09 \mathrm{t}(7.2)$ & $13.9 \mathrm{q}$ \\
\hline 9 & $2.20 \mathrm{dd}(4.8,7.2)$ & $47.0 \mathrm{~d}$ & $14-\mathrm{OCH}_{3}$ & $3.40 \mathrm{~s}$ & $57.8 \mathrm{q}$ \\
\hline 10 & $1.79 \mathrm{~m}$ & $43.3 \mathrm{~d}$ & $16-\mathrm{OCH}_{3}$ & $3.35 \mathrm{~s}$ & $56.4 \mathrm{q}$ \\
\hline 11 & - & $49.2 \mathrm{~s}$ & & & \\
\hline 12 & $\begin{array}{l}1.63 \mathrm{~m}(\alpha) \\
1.98^{a}(\beta)\end{array}$ & $29.9 \mathrm{t}$ & & & \\
\hline
\end{tabular}

${ }^{a}$ Overlapped signals.

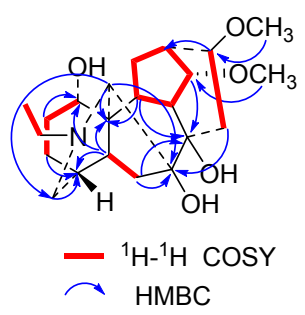

图 2 化合物 1 的关键 $\mathrm{HMBC}$ 与 ${ }^{1} \mathrm{H}-{ }^{1} \mathrm{H}$ COSY 相关 Figure 2 Key HMBC and ${ }^{1} \mathrm{H}^{-1} \mathrm{H}$ COSY correlations of compound 1

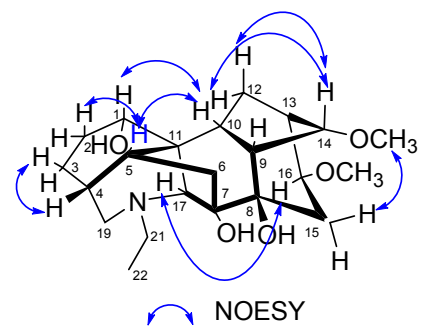

图 3 化合物 $\mathbf{1}$ 的关键 NOESY 相关

Fiture 3 Key NOESY correlations of compound 1

拒食活性进行研究报道. 另外, 在 $\mathrm{C}_{19}$ 二萜生物碱中, 化 合物 11 和 12 对甜菜夜蛾拒食作用较强 $\left(\mathrm{EC}_{50}<1 \mathrm{mg} /\right.$ $\mathrm{cm}^{2}$ ), 而 9 和 10 的作用相对较差 $\left(\mathrm{EC}_{50} \approx 7 \mathrm{mg} / \mathrm{cm}^{2}\right)$, 这 说明 C-18 位是否有邻氨基苯甲酰衍生物的取代对其活 性可能有较大影响. $\mathrm{C}_{20}$ 二萜生物碱 13 对甜菜夜蛾基本 没有活性 $\left(\mathrm{EC}_{50}>60 \mathrm{mg} / \mathrm{cm}^{2}\right)$.

\section{2 实验部分}

\section{1 仪器与试剂}

核磁共振仪(Brucker AVANCE DRX-400 和 600), 超 高效液相色谱仪(ACQUITY UPLC I-Class)与四级杆飞
表 2 白喉乌头中二萜生物碱对甜菜夜蛾的拒食活性

Table 2 Antifeedant activities of the diterpenoid alkaloids isolated from A. leucostomum against Spodoptera exigua Hiibner $(n=3)$

\begin{tabular}{cl||cc}
\hline Compd. & $\begin{array}{c}\mathrm{EC}_{50} /\left(\mathrm{mg} \bullet \mathrm{cm}^{-2}\right) \\
(95 \% \text { confidence limit })\end{array}$ & Compd. & $\begin{array}{c}\mathrm{EC}_{50} /\left(\mathrm{mg}^{\bullet} \mathrm{cm}^{-2}\right) \\
(95 \% \text { confidence limit })\end{array}$ \\
\hline $\mathbf{1}$ & $20.75(14.09,30.54)$ & $\mathbf{8}$ & $1.88(1.12,3.18)$ \\
$\mathbf{2}$ & $8.59(5.98,12.36)$ & $\mathbf{9}$ & $6.34(2.66,15.09)$ \\
$\mathbf{3}$ & $>30$ & $\mathbf{1 0}$ & $6.75(3.01,15.12)$ \\
$\mathbf{4}$ & $17.65(11.10,28.07)$ & $\mathbf{1 1}$ & $0.73(0.33,1.62)$ \\
$\mathbf{5}$ & $\mathrm{nt}^{a}$ & $\mathbf{1 2}$ & $0.84(0.49,1.45)$ \\
$\mathbf{6}$ & $(0.85,1.73)$ & $\mathbf{1 3}$ & $>60$ \\
7 & $1.45(0.75,2.81)$ & Aconitine & $0.02(0.01,0.11)$ \\
\hline${ }^{a}$ nt:
\end{tabular}

nt: not test (insufficient compound available).

行时间质谱仪(Xevo G2-S QTof) (waters 公司), 差示扫 描量热仪(Netzsch Jupiter STA 449C), 傅立叶红外光谱 仪 (Thermo Fisher Nicolet 6700), 自动精密旋光仪 (Perkin-Elmer 341). 层析用 YMC 反相硅胶填料, 层析 用氧化铝(上海陆都化学试剂厂), 层析用硅胶 $\mathrm{G}$ 和 $\mathrm{H}$ (青 岛海洋化工厂), 其余试剂为分析纯.

\section{2 实验样品}

白喉乌头于 2015 年 8 月采自于新疆伊犁尼勒克县, 由中国科学院华南植物园杨亲二研究员鉴定为白喉乌 头(Aconitum leucostomum Worosch.), 样品(No. C. Ren \& L. Wang 735)保存于中国科学院华南植物园.

\section{3 提取与分离}

干燥白喉乌头及地上部分 $(10 \mathrm{~kg})$ 粉碎后, $0.1 \mathrm{~mol} / \mathrm{L}$ 盐酸冷浸 6 次, 每次 $3 \mathrm{~d}$, 滤去残渣, 用饱和碳酸钠水溶 液调至中性, 再用氨水碱化至 $\mathrm{pH} 9 \sim 10$, 用 $\mathrm{CH}_{2} \mathrm{Cl}_{2}$ 萃 取, 每次 $5 \mathrm{~L}$, 共 4 次, 合并萃取液, 减压浓缩, 得总生 物碱(40 g). 
总生物碱 A $(40 \mathrm{~g})$ 经过硅胶柱层析 $[100 \sim 200$ 目硅 胶 $400 \mathrm{~g}$, 二氯甲烷-甲醇 (1\%二乙胺), $V: V=100$ ： $1 \rightarrow 0: 1]$ 梯度洗脱, 洗脱液经 $\mathrm{TLC}$ 检查合并为 $\mathrm{A}_{1} \sim \mathrm{A}_{4}$ 四个部分.

$\mathrm{A}_{1}$ 部分经硅胶柱层析[石油醚-丙酮 (1\%二乙胺), $V: V=60: 1 \rightarrow 4: 1]$ 洗脱得到组分 $\mathrm{A}_{1-1}$ 和组分 $\mathrm{A}_{1-2}$; 组 分 $\mathrm{A}_{1-1}$ 经硅胶柱层析[石油醚-丙酮( $1 \%$ 二胺), $V: V=$ $40: 1 \rightarrow 8: 1$ ] 洗脱得到化合物 $\mathbf{3}(22 \mathrm{mg})$ 和 $4(14 \mathrm{mg})$; 组 分 $\mathrm{A}_{1-2}$ 经硅胶柱层析[石油醚-丙酮( $1 \%$ 二胺), $V: V=$ $20: 1 \rightarrow 1: 1]$ 梯度洗脱得到 $\mathrm{A}_{1-2-1}$ 和 $\mathrm{A}_{1-2-2}$ 两个组分, $\mathrm{A}_{1-2-1}$ 组分用 [石油醚-丙酮(1\%二乙胺), $V: V=15: 1$ ] 洗 脱得化合物 $1(27 \mathrm{mg})$ 和 $2(15 \mathrm{mg})$; 反相硅胶柱层析(甲 醇一水, $V: V=1: 9)$ 分离组分 $\mathrm{A}_{1-2-2}$ 得化合物 $5(4 \mathrm{mg})$. $\mathrm{A}_{2}$ 部分经硅胶柱层析[石油醚-丙酮( $1 \%$ 二乙胺), $V: V=$ $40: 1 \rightarrow 1: 1$ ] 洗脱得 $A_{2-1}$ 和 $A_{2-2}$ 两个组分, $A_{2-1}$ 组分经硅 胶柱层析[石油醚-丙酮(1\%二乙胺), $V: V=15: 1 \rightarrow 1$ : 1]洗脱得化合物 $6(13 \mathrm{mg}$ ); 反相硅胶柱层析(甲醇-水, $V: V=6: 4)$ 分离组分 $\mathrm{A}_{2-2}$ 得化合物 $7(19 \mathrm{mg}) . \mathrm{A}_{3}$ 部分 经硅胶柱层析[石油醚-丙酮(1\%二乙胺), $V: V=20$ : $1 \rightarrow 1: 1$ ] 洗脱得到 $A_{3-1} \sim A_{3-3}$ 三个组分, 用石油梄丙酮 重结晶 $\mathrm{A}_{3-1}$ 得到化合物 $8(14 \mathrm{mg}) ; \mathrm{A}_{3-2}$ 部分经硅胶柱层 析[石油醚/丙酮(1\%二乙胺), $V: V=20: 1 \rightarrow 1: 1$ ]得化 合物 $9(12 \mathrm{mg})$ 和 $10(26 \mathrm{mg})$; 组分 $\mathrm{A}_{3-3}$ 经硅胶柱层析[石 油醚-丙酮(1\%二乙胺), $V: V=20: 1]$ 分离, 再用碱性氧 化铝柱层析(氯仿一甲醇, $V: V=15:$ 1)纯化得化合物 11 $\left(23 \mathrm{mg}\right.$ ); $\mathrm{A}_{4}$ 部分经硅胶柱层析 (二氯甲烷-甲醇, $V: V=$ $45: 0 \rightarrow 0: 1)$ 梯度洗脱得到 $\mathrm{A}_{4-1}$ 和 $\mathrm{A}_{4-2}$ 两个组分, $\mathrm{A}_{4-1}$ 用 反相柱层析(甲醇-水, $V: V=4: 1$ ) 得化合物 $12(22 \mathrm{mg}$ ), 用碱性氧化铝柱层析(氯仿-甲醇, $V: V=20: 1$ )分离组 分 $\mathrm{A}_{4-2}$ 得化合物 $\mathbf{1 3}(16 \mathrm{mg})$.

leucostonine (1): 白色固体. m.p. $258.6 \sim 259.1{ }^{\circ} \mathrm{C}$; $[\alpha]_{\mathrm{D}}^{20}-24\left(c 0.30, \mathrm{CHCl}_{3}\right) ;{ }^{1} \mathrm{H}$ NMR $\left(400 \mathrm{MHz}, \mathrm{CDCl}_{3}\right)$ 及 ${ }^{13} \mathrm{C}$ NMR (100 MHz, $\mathrm{CDCl}_{3}$ ) 数据见表 1; IR (KBr) $v_{\max }$ : 3392, 2934, 2819, 2671, 1631, 1602, 1492, 1463, 1384, 1347, 1329, 1299, 1214, 1118, 1098, 1062, 1033, 1010, 997, 978, $961 \mathrm{~cm}^{-1}$. HR-ESI-MS calcd for $\mathrm{C}_{22} \mathrm{H}_{36} \mathrm{NO}_{5}$ $[\mathrm{M}+\mathrm{H}]^{+}$394.2593, found 394.2584.

\section{4 拒食活性}

甜菜夜蛾, 在温度为 $(27 \pm 1){ }^{\circ} \mathrm{C}$, 湿度为 $70 \%$ $80 \%$, 光周期为 $14: 10 \mathrm{~h}(\mathrm{~L}: \mathrm{D})$ 的条件下饲养, 挑选虫 体均匀一致、健壮并饥饿 $6 \mathrm{~h}$ 的 3 龄幼虫进行试验.

采用 González-Coloma 等 ${ }^{[17]}$ 推荐的选择性叶碟法进 行活性测定: 用打孔器将新鲜甘蓝叶片裁成直径 $2 \mathrm{~cm}$ 的叶碟, 在表面均匀涂上 $15 \mu \mathrm{L}$ 药液(处理组)或空白液 (对照组). 分别将两片对照组和处理组叶片放置于直径
为 $15 \mathrm{~cm}$ 的培养血中, 待液体风干后, 每血放入 6 头饥 饿 $6 \mathrm{~h}$ 的幼虫. 每个处理重复三次. $24 \mathrm{~h}$ 后用方格纸片法 测定取食面积，并用以下公式计算拒食率: $\mathrm{FR} \%=(\mathrm{CK}$ $-T) / C K \times 100 \%$. 其中 $\mathrm{CK}$ 为对照组叶片取食面积, $T$ 为 药剂处理组叶片取食面积.

对于 $\mathrm{FR}>50 \%$ 的化合物测试其 $\mathrm{EC}_{50}$ 值(引起 $50 \%$ 个 体有效的剂量). $\mathrm{EC}_{50}$ 值测定方法: 依据初篎的浓度, 采 用梯度稀释法配制一系列浓度的药液, 按照前述方法进 行试验, 测定取食面积, 计算拒食率, 并通过线性回归 曲线计算相应的 $\mathrm{EC}_{50}$ 值.

辅助材料(Supporting Information) IR, HR-ESI-MS, 1D NMR 和 2D NMR 图谱, 这些材料可以免费从本刊网 站(http://sioc-journal.cn/)上下载.

\section{Referenes}

[1] Xiao, P.-G.; Wang, F.-P.; Gao, F.; Yan, L.-P.; Chen, D.-L.; Liu, Y. Acta Phytotaxon. Sin. 2006, 44, 1 (in Chinese).

(肖培根, 王锋鹏, 高峰, 问路平, 陈东林, 刘勇, 植物分类学报, 2006, 44, 1.)

[2] Institute of Botany, Chinese Academy of Sciences and Institute of Materia Medica, Chinese Academy of Medical Sciences, Flora Reipublicae Populais Sinica, Vol. 27, Science Press, Beijing, 1979, p. 171 (in Chinese).

(中国科学院植物所, 中国医学科学院药物所, 中国植物志, 科 学出版社, 北京, 1979, Vol. 27, p. 171.)

[3] (a) Xu, W.-L.; Chen, C.; Shan, L.-H.; Gao, F.; Huang, S.; Zhou, X.-L. Heterocycles 2016, 92, 2059.

(b) Yue, J.-M.; Xu, J.; Zhao, Q.-S.; Sun, H.-D.; Chen, Y.-Z. J. Nat. Prod. 1996, 59, 277.

(c) Telnov, V. A.; Yunusov, M. S.; Abdullaev, N. D.; Zhamierashvili, M. G. Khim. Prir. Soedin 1988, 4, 556.

(d) Wei, X.-Y.; Wei, B.-Y.; Zhang, J. Acta Bot. Sinica 1996, 38, 995.

[4] Shan, L.-H.; Zhang, J.-F.; Chen, L.; Wang, J.-X.; Huang, S.; Zhou, X.-L. Nat. Prod. Commun. 2015, 10, 2067.

[5] Chen, L.; Shan, L.-H.; Xu, W.-L.; Zhang, J.-F.; Huang, S.; Zhou, X.-L. Nat. Prod. Res. 2017, 31, 523.

[6] Sayed, H. M.; Desai, H. K.; Ross, S. A., Pelletier, S. W.; Aasen, A. J. J. Nat. Prod. 1992, 55, 1595.

[7] Wei, X.-Y.; Wei, B.-Y.; Zhang, J. Acta Bot. Sin. 1996, 38, 995 (in Chinese).

(魏孝义，韦璧瑜，张继，植物学报，1996, 38, 995.)

[8] Peng, C.-S.; Wang, J.-Z.; Jian, X.-X.; Wang, F.-P. Nat. Prod. Res. Dev. 2000, 12, 45 (in Chinese).

(彭崇胜, 王建忠, 简锡贤, 王锋鹏, 天然产物研究与开发, 2000, 12, 45.)

[9] Ross, S. A.; Pelletier, S. W.; Aasen, A. J. Tetrahedron 1992, 48, 1183.

[10] Jiang, S.-H.; Zhu, Y.-L.; Zhu, R.-H. Acta Pharm. Sin. 1982, 17, 288 (in Chinese).

(蒋山好, 朱元龙, 朱任宏, 药学学报, 1982, 17, 288.)

[11] De la Fuente, G.; Reina, M.; Valencia, E.; Rodriguez-Ojeda, A. Heterocycles 1988, 27, 1109.

[12] Vaisov, Z. M.; Bessonova I. A. Chem. Nat. Compd. 1992, 28, 463.

[13] Yunusov, M. S.; Yunusov, S, Y. Chem. Nat. Compd. 1970, 6, 334.

[14] Wei, X.-Y.; Wei, B.-Y.; Zhang, J. Chin. Tradit. Herbal Drugs 1995, 26, 344 (in Chinese)

(魏孝义，韦璧瑜，张继，中草药, 1995, 26, 344.)

[15] Hao, Z.-G.; Liu, J.-H.; Zhao, S.-X.; Miao, Z.-C. Phytochemistry 1991, 30, 3494. 
[16] Feng, F.; Liu, W.-Y.; Chen, Y.-S.; Ye, W.-C.; Liu, J.-H.; Zhao, S.-X. J. Chin. Pharm. Univ. 2003, 34, 17 (in Chinese).

(冯锋, 柳文媛, 陈优生, 叶文才, 刘静涵, 赵守训, 中国药科大 学学报, 2003, 34, 17.)
[17] (a) Gonzalez-Coloma, A.; Reina, M.; Cabrera, R.; Castañera, P.; Gutierrez, C. J. Chem. Ecol. 1995, 21, 1255.

(b) González-Coloma, A.; Terrero, D.; Perales, A.; Castañera, P.; Fraga, B. M. J. Agric. Food Chem. 1996, 44, 296.

(Lu, Y.) 